\title{
Spatiotemporal Nonlinear Beam Shaping
}

\author{
S. Wabnitz ${ }^{1, *}$, K. Krupa ${ }^{2,3}$, A.Tonello ${ }^{2}$, A. Bendahmane ${ }^{3}$, R. Dupiol ${ }^{3}$, B. M. Shalaby ${ }^{2}$, \\ M. Fabert ${ }^{2}$, Ch. Louot ${ }^{2}$, R. Guenard ${ }^{2}$, A. Barthélémy ${ }^{2}$, G. Millot ${ }^{3}$, V. Couderc ${ }^{2}$ \\ 1 Dipartimento di Ingegneria dell'Informazione and INO-CNR, Università di Brescia, via Branze 38, 25123, Brescia, \\ Italy \\ 2 Université de Limoges, XLIM, UMR CNRS 7252, 123 Avenue A. Thomas, 87060 Limoges, France \\ 3 Université de Bourgogne Franche-Comté, ICB UMR CNRS 6303, 9 Avenue A. Savary 21078 Dijon, France \\ *stefan.wabnitz@unibs.it
}

\begin{abstract}
The reshaping of multimode waves in optical fibers is a process where the spatial and spectral degrees of freedom are inherently coupled. Our experiments demonstrate that pumping a graded-index multimode fiber with sub-ns pulses from a microchip Nd:YAG laser leads to supercontinuum generation with a uniform bell-shaped spatial beam profile.
\end{abstract}

OCIS codes: (060.4370) Nonlinear optics, fibers, (320.6629) Supercontinuum generation, (190.4410) Nonlinear optics, parametric processes

\section{Introduction}

The strong mode confinement and the versatile dispersion engineering of single mode photonic crystal fibers have enabled, over the past two decades, efficient and spatially coherent supercontinuum (SC) generation sources spanning from the ultra-violet to the mid-infrared. However, because of their small mode area, single mode optical fibers are of limited use for applications where high powers are required, such as fiber lasers and beam delivery. For this reason, it would be very interesting to use large area, multimode fibers (MMFs) for substantially extending the power range of SC light sources. On the other hand, it is well known that MMFs such as graded-index (GRIN) fibers that support the propagation of 100-1000 guided modes are subject to strong mode-mixing, that spoils the initial spatial coherence of a light beam after a few mm of length. This is a serious drawback in applications where preservation of spatial coherence is required.

In this talk, we report on our recent striking discovery that the cubic, or Kerr, nonlinearity of glass fibers may lead to the formation of a spatially coherent nonlinear beam in a highly multimode GRIN fiber [1]. In other words, linear mode mixing is effectively suppressed at high optical powers, and a light beam is self-guided, in analogy with the mechanism that leads to the formation of a fundamental spatial optical soliton, or to the effect of wave condensation [2]. It is important to note that such Kerr-induced nonlinear guiding occurs for power levels which are at least one order of magnitude lower than the value required for the well-known effect of catastrophic light self-focusing in a GRIN MMF [3]. Moreover, the nonlinear recovery of spatial coherence or Kerr self-cleaning occurs, first of all, at the wavelength of the pump beam: it is thus fundamentally different from the well-known Raman induced beam clean-up that is observed at the Stokes wavelength [4].

\section{Spatio-temporal nonlinear coupling}

In stark contrast with the case of single mode fibers, where SC generation is a purely temporal phenomenon, nonlinear spectral broadening in MMFs is a result of a complex interplay between the spatial and the temporal degrees of freedom. In fact, multimode beams in a GRIN MMF undergo a periodic beating (or self-imaging) along the longitudinal direction [5]: in the presence of the Kerr effect, a nonlinear grating results. This dynamic grating permits the quasi-phase-matching of dispersive wave sidebands shed by temporal multimode solitons in the anomalous dispersion regime [6], and by quasi-CW pulses in the normal dispersion regime [7]. As a result, an array of intense sidebands is observed, that extends over multiple octaves, irrespective of the sign of chromatic dispersion at the pumping wavelength [6-8].

The next question naturally arises, namely is the nonlinear recovery of spatial coherence a property which is only enjoyed by the pumping beam, or is spatial coherence transferred across the array of frequency-shifted sidebands as well? Although in general the answer strongly depends on the input launching condition of the pump beam into the MMF, quite remarkably, we found that the response to this important question may be affirmative. Namely, by 

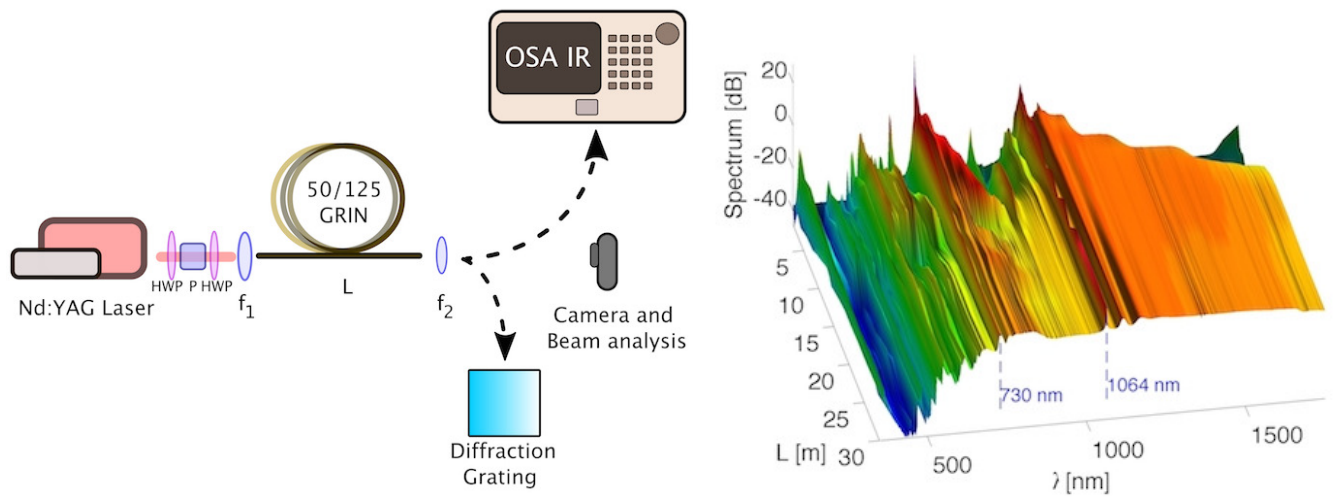

Fig. 1. Left: Experimental setup. HWP: half-wave plate; P: cube polarizer; $f_{1}, f_{2}$, lenses; Nd:YAG laser: $900 \mathrm{ps}, 30 \mathrm{kHz}, 1.4 \mathrm{~W}$ average power in the fiber. Right: experimental results of the cut-back technique (input average power in the fiber $1.4 \mathrm{~W}$, drops to $670 \mathrm{~mW}$ after $30 \mathrm{~m}$ of fiber). All experimental spectra have been visualized by normalizing to the level at $1118 \mathrm{~nm}$.

separately filtering different sidebands across the entire spectrum from the visible into the near-infrared, we found that it is possible to observe that the coherent bell-shaped spatial profile is maintained [7].

For many applications, it is desirable to use a SC light source with a broad and flat spectral intensity profile. We found that, as the pump power grows larger (while still remaining well below the self-focusing threshold), the spectral sidebands merge until a bright SC is generated, that exhibits a remarkable degree of spectral flatness. In addition, nonlinear spatial coherence is now maintained across the entire spectral bandwidth of the SC. The cleaning effect in the infrared part of the SC is also enforced by the cooperative action of Raman scattering. In the next section, we present our recent original experimental results that demonstrate the generation of a spatially coherent SC in a GRIN MMF. In particular, by means of cut-back measurements, we could study the spectral development of SC along the fiber.

\section{Supercontinuum self-cleaning}

In our experiments, as shown in Fig. 1, left, we used as pump source an amplified Nd:YAG microchip laser, delivering 900 ps pulses at $1064 \mathrm{~nm} 30 \mathrm{kHz}$ repetition rate. The polarized Gaussian pump pulses were launched into the GRIN MMF by using a lens with focal length of $f_{1}=50 \mathrm{~mm}$ and a three-axis translating stage. At the input face of the fiber the beam had a FWHMI diameter of $40 \mu \mathrm{m}$, which is close to the fiber core diameter.

We used an optical spectrum analyzer covering the spectral range from $350 \mathrm{~nm}$ up to $1750 \mathrm{~nm}$. The beam profile (at the output face of the fiber) was imaged on a CCD camera through an $f_{2}=8 \mathrm{~mm}$ microlens. Different $10 \mathrm{~nm}$-wide bandpass interference optical filters were introduced to analyze the spatial dynamics at the pump wavelength and other wavelengths across the SC. Note that, owing to the long pump pulse duration and the low modal GRIN MMF dispersion, a large number of initially excited modes maintain their temporal superposition and nonlinear coupling over tens of meters.

In Fig. 1, right, we display the result of cut-back experiments which show the development of the SC along the length of the GRIN MMF. As can be seen, a remarkable spectral flatness is obtained at the near infrared side of the SC spectrum. The high spectral peak at about $730 \mathrm{~nm}$ corresponds to the first-order anti-Stokes Longhi sideband $[5,7]$.

The detail of the longitudinal evolution of SC is provided by the left plot of Fig. 2, where we compare the growth along the GRIN MMF of a selected series of spectral components. While some components of the visible spectrum (such as the component at $730 \mathrm{~nm}$ ) as well as the spectral broadening until $1142 \mathrm{~nm}$ occur over the first meter of fiber, the spectral components around $1600 \mathrm{~nm}$ only grow after about $8 \mathrm{~m}$ of MMF.

The effect of SC spatial self-cleaning is demonstrated by the right plots of Fig.2, which display the far field that emerges from the MMF at selected wavelengths across the entire spectrum. As can be seen, for all wavelengths a bell-shaped spatial profile is observed. At those fiber lengths the SC is also enforced by the presence of the Raman effect, which privileges bell-shaped beams. However similar results for near and far fields (here not shown) were also observed in the visible domain and at the pump wavelength for fiber lengths shorter than $8 \mathrm{~m}$, that is well before the 

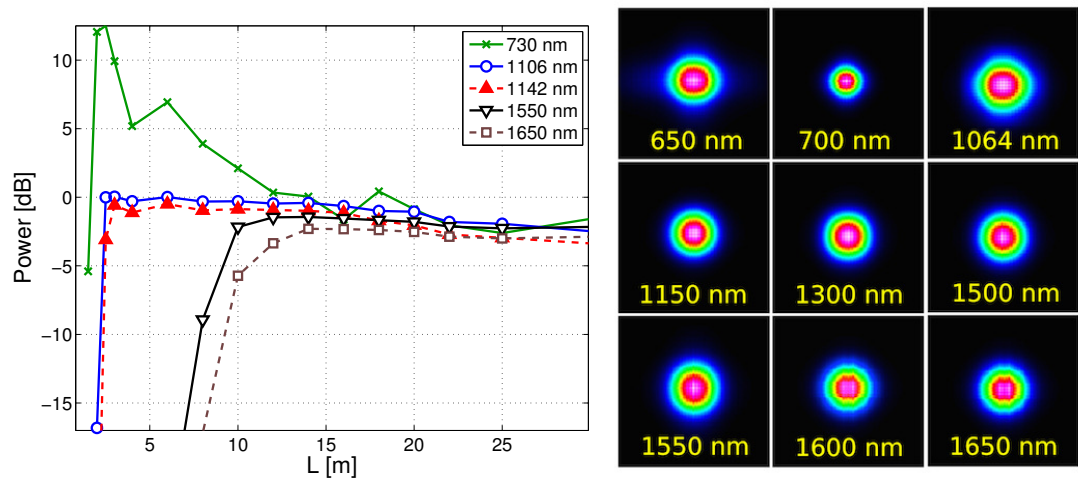

Fig. 2. Left: detail of spectral intensities at different wavelengths. Right: far field at the output for $\mathrm{L}=30 \mathrm{~m}, 1.4 \mathrm{~W}$ input average power using bandpass filters (10 $\mathrm{nm}$ bandwidth) with different center wavelengths.

onset of strong Raman conversion [7].

\section{Conclusions}

We have discussed how multimode fibers provide a new, intriguing and low-cost platform for SC generation, which enables much higher spectral brightness when compared with single mode fibers. At the same time, we have demonstrated that pumping a GRIN MMF with a sub-nanosecond microchip laser in the normal dispersion regime of a MMF permits to maintain the spatial coherence of the SC, thanks to the Kerr self-cleaning effect.

\section{References}

1. K. Krupa, A. Tonello, B. M. Shalaby, M. Fabert, A. Barthélémy, G. Millot, S. Wabnitz, and V. Couderc , "Spatial beam self-cleaning in multimode fiber," arXiv:1603.02972 (2016).

2. P. Aschieri, J. Garnier, C. Michel, V. Doya, and A. Picozzi, "Condensation and thermalization of classical optical waves in a waveguide," Phys. Rev. A. 83, 033838 (2011).

3. J.T. Manassah, P.L. Baldeck, and R.R. Alfano, "Self-focusing and self-phase modulation in a parabolic gradedindex optical fiber," Opt. Lett. 15, 589-591 (1988).

4. N. B. Terry, T. G. Alley, and T. H. Russell, "An explanation of SRS beam cleanup in graded- index fibers and the absence of SRS beam cleanup in step-index fibers," Opt. Express 15, 17509-17519 (2007).

5. S. Longhi, "Modulational instability and space-time dynamics in nonlinear parabolic-index optical fibers," Opt. Lett. 28, 2363-2365 (2003).

6. L. G. Wright, S. Wabnitz, D. N. Christodoulides, and F. Wise, "Ultra-broadband dispersive radiation by spatiotemporal oscillation of multi-mode waves," Phys. Rev. Lett. 115, 223902 (2015).

7. K. Krupa, A. Tonello, A. Barthélémy, V. Couderc, B. M. Shalaby, A. Bendahmane, G. Millot and S. Wabnitz, "Observation of Geometric Parametric Instability Induced by the Periodic Spatial Self-Imaging of Multimode Waves,” Phys. Rev. Lett. 116, 183901 (2016).

8. L. G. Wright, D. N. Christodoulides, and F. Wise, "Controllable spatiotemporal nonlinear effects in multimode fibres," Nature Photonics 9, 306-310 (2015). 